\title{
Linguistic senvices in healthcare sector as factor in access to medical care - Bulgarian perspective
}

\author{
Alexandrina Vodenitcharova \\ Faculty of Public Health, Medical University - Sofia
}

\section{Abstract}

The lack of adequate linguistic services in healthcare could result violations or lack of attention to human rights, which can have serious consequences. Such condition can even cause discrimination in the delivery of health services and violation to fundamental human rights.

Bulgarian national legislation only contains provisions, which set up a responsibility for the treating physician to keep a medical file of the patient, but does not introduce strict obligation for healthcare providers to maintain language services to patients who cannot access the national language.

It is crucial for patients to have full access to information in the course of providing medical care, because this is the only way to be guaranteed the patients' autonomy.

\section{Keywords: Healthcare providers, linguistic services, access, medical care}

Health systems around the world differ form each other, as they reflect different societal choices. The health systems in the European Union are a central component of the high levels of social protection, and contribute to social cohesion and social justice as well as to sustainable development.

European Union health systems increasingly interact with each other and especially after 2011 when a Directive 2011/24/EU on the application of patients' rights in cross-border healthcare was first introduced (1).

European health systems face growing common challenges: increasing cost of healthcare, matters associated with population ageing, rise of chronic diseases and multi-morbidity, as factors leading to growing demand for healthcare. Another challenges faced by European health systems include shortages and uneven distribution of health professionals, health inequalities and inequities in access to healthcare. 
The possibility for patients to receive cross-border healthcare under Directive 2011/24/EU retain to EU Member States the responsibility for providing safe, high quality, efficient and quantitatively adequate healthcare to citizens on their territory. This responsibility could be provided equally to all EU citizens, only if patients could express freely their informed consent for obtaining medical services. Challenges are faced in circumstances where patient and healthcare providers do not share the same language and where adequate linguistic support is not provided to patients by the healthcare provider.

The lack of adequate linguistic services in healthcare could result violations or lack of attention to human rights, which can have serious consequences. Such condition can even cause discrimination in the delivery of health services and violation to fundamental human rights (2).

In relation to European Union regulatory framework on cross-border healthcare services, there is a lack of provisions directly addressing to language services for EU citizens or legal residents pursuing healthcare in Member States where they cannot access the information. In such situation, it is up to every European Union Member State to decide what kind of regulation will be introduced in order to facilitate the access to information by patients receiving medical services in another EU country.

In Bulgaria, it is not evident the introduction of legislation that contemplates the provision of language services to patients who cannot access the national language. Healthcare providers in Bulgaria are not strictly obliged by the law to provide professional language support.

National legislation only contains provisions, which set up a responsibility for the treating physician to keep a medical file of the patient. A definition of the term "health information" is included in the legislation, under the meaning of "the personal data related to the health condition, the physical and mental development of individuals, as well as any other information contained in medical prescriptions, instructions, protocols, certificates and other medical documentation" (3).

Patient rights regarding the medical file are regulated in both specific and general national legal acts. Specific regulation could be found in the Health Act and the Professional Ethics Code, while general rules on medical files could be found in the Personal Data Protection Act.

In order for patients to obtain the necessary medical information, sometimes ad-hoc language brokers are called to perform translation, interpreting and language/cultural mediation.

In cases when healthcare providers do not offer professional linguistic services, family members of the patient, as well as bilingual staff or volunteers translate and interpret the necessary information on. This is not a convenient solution, though, because commonly it is not obvious that bilingual staff in medical facilities, who have been invited for translation/interpretation, obtain a verification document for the degree of proficiency in both languages. That is the reason why it is not always clear that bilingual staff is capable of providing duties of translator or interpreter.

It is not a rare situation when cross-border patients are responsible for the translation of documents and medical records.

Access to healthcare services and medical care is directly related to the opportunity to consent a patient (4). When there is lack of understanding of the information provided to the patient, there is a great risk the 
obtained consent not to be valid, since the informed consent is a process by which the treating health care provider discloses appropriate information to a competent patient so that the patient may make a voluntary choice to accept or refuse treatment.

It is crucial for patients to have full access to information in the course of providing medical care, because this is the only way to be guaranteed the patients' autonomy. Healthcare providers must guarantee to their patients full access to information, regardless of the language in which information is provided.

The lack of proper regulatory framework both on European union level and on national level in Bulgaria, need to be overcome by taking appropriate measures, like it has been regulated in cases of citizens who do not speak the societal language regarding access and communication in legal matters, specifically concerning criminal proceedings.

When being introduced, as part of Bulgarian national legal framework, the obligation of healthcare providers to provide linguistic services to their patients in order to guarantee full access to information, this will also facilitate the maintenance of high-quality of healthcare services. By introducing this approach, a high-quality healthcare system will ensure that everyone within the system has access to appropriate care. Introducing linguistic services by healthcare providers will have significant impact to health outcomes as it will reduce health inequalities and will provide high-quality healthcare services for benefit of the individuals and the society.

\section{References}

1. Directive 2011/24/EU of the European Parliament and of the Council of 9 March 2011 on the application of patients' rights in cross-border healthcare, OJ L 88, 4.4.2011, pp. 45-65

2. European Commission, Studies on translation and multilingualism. Public service translation in cross border healthcare, 2015.

3. Health Law, Published in State Gazette, issue 70 of August 10th 2004.

4. Kehayov, A. Modern approach in exercises the right to health care, Medinform, 2016, 3(2), 484-487.

\section{Corresponding author:}

Assist. Prof. Alexandrina Vodenitcharova, PhD

Faculty of Public Health

Medical University - Sofia

1527 Sofia, Bulgaria

8 Bialo more str.

E-mail: a.vodenicharova@foz.mu-sofia.bg 